\title{
FPS Overseas Consultants
}

\section{A Preliminary List}

THE FPS is forming a panel of overseas consultants and correspondents to keep the Society informed and in touch with conservation work, problems and progress overseas. This is a preliminary list of those who have so far accepted our invitation, and to whom we are most grateful for their willingness to serve:

\section{AFRICA}

Ethiopia . . John Blower, Senior Game Warden.

Kenya . . Leslie Brown.

Michael Sawyer, Chief Executive, East African Wild Life Society.

Madagascar . Dr. J.-J. Petter, Sous-Directeur, Muséum National d'Histoire Générale.

Malawi . . G. D. Hayes, Hon. Secretary, Fauna Preservation Society of Malawi.

South Africa . Dr. R. Bigalke, Deputy President, Wild Life Protection and Conservation Society of South Africa.

Mrs. C. Niven.

Sudan . . M. K. Shawki, Director General, Rural Water and Development Corporation.

Tanzania . . Dr. Hugh Lamprey, Director, Serengeti Research Institute.

Uganda . . Roger Wheater, Warden, Murchison Falls National Park.

Zambia . . Lt. Col. R. A. Critchley, DSO, MC, President, Wild Life Conservation Society of Zambia.

\section{ASIA}

Burma . . U Tun Yin.

India . . Zafar Futehally, Hon. Secretary, Bombay Natural History Society.

E. P. Gee, member, Indian Wild Life Board.

Ceylon . . C. E. Norris, lately President, Wild Life Protection Society of Ceylon.

Indonesia . . Dr. Hasan Basjarudin, Director, Forest Service. Iran . . Eskandar Firouz, Secretary General, Game and Fish Department.

Israel . . Dr. A. Zahavi, Hon. Secretary, Society for the Protection of Nature in Israel.

Malaysia . . Dr. E. Balasingam, President, Malayan Nature Society.

Lord Medway, Zoology Department, University of Malaya. 


\section{AUSTRALASIA}

Australia
Graham Pizzey, Victoria.

Harry Shugg, Fauna Officer, Western Australia.

NORTH AMERICA

Bermuda

David B. Wingate, Museum and Conservation Officer.

Canada . . Professor A. de Vos.

Patrick Hardy, Managing Director and Editor, Canadian Audubon Society.

J. Livingston, Canadian Broadcasting Corporation.

USA . . Roland Clement, Vice-President, National Audubon Society.

Dr. Roger Tory Peterson.

Richard H. Pough, President, Natural Area Council.

Dr. Lee M. Talbot, Smithsonian Field Representative for International Affairs in Ecology and Conservation, Museum of Natural History.

Judge Russell Train, Chairman, Conservation Foundation.

\section{SOUTH AMERICA}

Brazil

Dr. Maria Buchinger, Latin American Desk, Nature Conservancy, Washington, DC.

Rio de Janeiro.

Colombia . . Dr. F. C. Lehmann, Director General, Valle State Museum of Natural History, Cali.

Peru . . Major Ian Grimwood, Wildlife Adviser, Peruvian Government.

\section{EUROPE}
Austria
Dr. H. Freundl, Hon. Secretary, World Wildlife Fund National Appeal. Department, Institut Royal des Sciences Naturelles.
Belgium . . E. Kesteloot, Head of Nature Conservation
Germany . . Professor Bernhard Grzimek, Director, Frank- furt Zoo.
Ireland . . Fergus O'Gorman, Department of Lands.
Italy . . Professor A. Toschi.
Netherlands . Dr. C. Maliepaard.
Sweden . . Dr. K. Curry-Lindahl, Director, Zoological De- partment, Nordiska Museet and Skansen.
USSR . . . Dr. A. Bannikov, All-Russian Society of the Protection of Nature. 